\title{
Estimation of Reproduction Numbers for the Period of the COVID-19 Epidemic Outbreak in the Central African Republic
}

\author{
Brice M. Yambiyo' ${ }^{1,2}$, A. Manirakiza², Gaston M. N'Guérékata ${ }^{3}$ \\ ${ }^{1}$ NEERLab, Department of Mathematics and Computer Science, Université de Bangui, Bangui, Central African Republic \\ ${ }^{2}$ Institut Pasteur Bangui Epidemiological Service, Rue Louis Pasteur (RCA), Bangui, Central African Republic \\ ${ }^{3}$ NEERLab, Morgan State University, Baltimore, MD, USA \\ Email: ybricemartial@gmail.com, amanirak@yahoo.fr, Gaston.N'Guerekata@morgan.edu
}

How to cite this paper: Yambiyo, B.M., Manirakiza, A. and N'Guérékata, G.M. (2021) Estimation of Reproduction Numbers for the Period of the COVID-19 Epidemic Outbreak in the Central African Republic. Applied Mathematics, 12, 477-488.

https://doi.org/10.4236/am.2021.126033

Received: April 8, 2021

Accepted: June 25, 2021

Published: June 28, 2021

Copyright $\odot 2021$ by author(s) and Scientific Research Publishing Inc. This work is licensed under the Creative Commons Attribution International License (CC BY 4.0).

http://creativecommons.org/licenses/by/4.0/

\begin{abstract}
In this paper, we propose a susceptible-exposed-infection-asymptomatichospitalized-recovered (SEIAHR) model with parameters on retrospective social distancing and masking. We estimated the model parameters from information published on the World Health Organization (WHO) website. We found that the actual reproduction number $R_{t}$ varies over the period from 03 March to 07 June 2020 and moreover, effective control over contacts and the frequency of population movement would reduce the evolution of the epidemic (control $c \geq 50 \%$ ). And the contact check has an influence on the base reproduction number $R_{0}$.
\end{abstract}

\section{Keywords}

Modeling, Mathematical Epidemiology, Data Analysis

\section{Introduction}

COVID-19 is an infectious disease caused by the latest coronavirus (SARS-CoV -2) which outbroke on November 17, 2019 in Hubei province (in central China), more precisely in the city of Wuhan. On March 11, 2020, the COVID-19 epidemic is declared a pandemic by the World Health Organization (WHO), which calls for essential protective measures to prevent the saturation of intensive care services and also to strengthen preventive hygiene measures (elimination of physical contact, kisses and handshakes, etc.).

In the Central African Republic, surveillance for the COVID-19 epidemic began from March 3, 2020. This surveillance concerns travelers coming from countries (France, Italy and China) affected by this epidemic. After the confir- 
mation of the first case imported from Italy on March 14, 2020, and some sporadic cases, the government through the Ministry of Health decided that all people coming by the different routes of entry and exit of the country are systematically screened.

School closures followed, removal of gatherings of more than 15 people, and barrier measures applied. It should also be noted that the population was very resentful about the barrier measures due to the habit of over a decade of greeting with handshake, kisses and gatherings during major celebrations (marriage, mourning, baptism, etc.). The government has therefore taken great measures to educate the population about the COVID-19 epidemic.

Pioneers Kermack and McKendrick made mathematical models gain much more attention today. Usually, models included three conditions in the total population: S for susceptible, I for infected, and R for cured, for example, L. Allen in [1] to study the positivity of solutions in the case of an SIS model. Likewise, Y. Cai et al. in [2] introduced the stochastic form of the SIS model incorporating media coverage to study the effects of environmental fluxes. Also, B. M. Yambiyo et al. studied the SIR model using the fractional derivative of Caputo. They studied the stability of solutions using the homotopy pertubation technique [3].

Some pathologies take into account exposure to the disease, which means that the population is in this case divided into four (4) compartments ( $\mathrm{S}$ for susceptible, E for exposed, I for infectious and $\mathrm{R}$ for recovered), more details in [4] [5] [6].

Adam Kucharski in [7], they developed the same type of model (SEIR). They took into account the delays in the onset and reporting of symptoms, thereby including compartments to reflect transitions and reporting states and disease states in Wuhan.

Abhijit Majumder1 et al. [8], used another type of epidemic model, notably the stochastic model, to determine the end point of disease extinction. They also adjusted the epidemic data for Italy and estimated the epidemic burden for that country.

Jonathan M. Read et al. [9], estimated the baseline reproduction number to be 3.11 (95\% CI, 2.39 - 4.13). It is therefore $58 \%$ to $76 \%$ of transmissions that must be prevented to stop increasing; finding cases in Wuhan of 5.0\% (3.6 - 7.4); 21,022 $(11,090$ - 33,490) total infections in Wuhan from January 1 to 22, 2020.

On the other hand, certain diseases such as hepatitis B, hepatitis C, AIDS and today COVID-19, the exposed host $\mathrm{E}$ plays a very important role when dynamic behaviors are expected to be discussed.

Recent literature [10] [11] has shown that a model of the Susceptible-Exposed-Infected-Recovered (SEIR) type is more suitable for studying the dynamics of COVID-19.

In the world, in order to understand the dynamics and this pandemic, several mathematical models in epidemiology have been developed, for more details on 
these different models in [12] [13] [14] [15] and the references within for much more details.

The spread and control of infectious diseases have been described by mathematical models, in order to study the dynamic properties of the disease.

We used an extension of the model (SEIR) by including asymptomatic infected and symptomatic infected compartments after being infected. The use of this extension comes from the reality of the dynamics of transmission of COVID-19 between 03 March 2020 and 07 June 2020 in the Central African Republic. We have found that when a person is exposed, they can become infected and transmit the disease whether asymptomatic or symptomatic. However, we do not take into account the delay in onset of symptoms since it varies from person to person.

In our work, we propose an SEIR model extended to nonlinear incidence with evaluation of social distancing on the dynamics of COVID-19 in order to estimate the effective number of $R_{t}$ reproduction in the Central African Republic.

\section{Mathematical Model}

Compared to the epidemiological reality of the Central African Republic concerning this pandemic disease of COVID-19, our model is as follows:

$$
\left\{\begin{array}{l}
\frac{\partial S(t)}{\partial t}=\Lambda-\beta S(t) \frac{q E(t)+I(t)+A(t)}{N(t)} \\
\frac{\partial E(t)}{\partial t}=\beta S(t) \frac{q E(t)+I(t)+A(t)}{N(t)}+\varepsilon E(t) \\
\frac{\partial I(t)}{\partial t}=p \varepsilon E(t)-\left(\gamma_{1}+\tau\right) I(t) \\
\frac{\partial A(t)}{\partial t}=(1-p) \varepsilon E(t)-\gamma_{2} A(t) \\
\frac{\partial H(t)}{\partial t}=p \varepsilon E(t) \\
\frac{\partial R(t)}{\partial t}=\left(\gamma_{1}+\tau\right) I(t)+\gamma_{2} A(t)
\end{array}\right.
$$

\section{Biological interpretation of parameters:}

- $N(t)=S(t)+E(t)+I(t)+H(t)+R(t)$ the total sum of the population at time $t$;

- $\beta$ Average transmission rate;

- $q=(1-c)$ transmissibility reduction factor of the exposed class where $c$ corresponds to the control intensity;

- $\Lambda$ recruitment rate of the population;

- $\varepsilon$ exposure rate;

- $\gamma_{1}$ infection rate of symptomatic;

- $\gamma_{2}$ infection rate of asymptomatic;

- $\tau$ COVID-19 death rate. 


\subsection{Population of Susceptible $S(t)$}

The sensitive population is increased by the net influx of recruitment $\Lambda$ of individuals and is decreased by natural death (which we neglect over the study period and therefore is not part of our model).

The susceptible population also decreases following an infection, acquired by contact between a susceptible person and an infected person, which can be symptomatic, asymptomatic. The transmission coefficients for these classes of infected individuals are respectively $\beta$ and $q$. In this paper, we use a basic transmission coefficient, $\beta$ (which models both the infectivity of COVID-19 and contact rates), with modifying factors for asymptomatic individuals $q$.

\subsection{Exposure Population $E(t)$}

These are individuals exposed to the COVID-19 virus, but there is no evidence that they are infected and can be infected asymptomatic or symptomatic.

\subsection{Infected $I(t)$}

These are individuals exposed to the virus and who subsequently became infected and confirmed in the laboratory. They are therefore either asymptomatic and symptomatic. The symptomatic population is generated after the development of clinical symptoms of COVID-19 by members of the exposed class.

$0<p \leq 1$ proportion of exposed individuals progresses to infectious class $I(t)$ at a rate $\mathrm{e}$ and the rest $(1-p)$ goes to the asymptomatic class.

\subsection{Asymptomatic $A(t)$}

These are laboratory confirmed and infected individuals, but have not developed clinical signs related to COVID-19. This is the proportion $(1-p)$ of infected presentations that progressed to the asymptomatic class.

\subsection{Hospitalized Serious Symptomatic and Notified $H(t)$}

This is the proportion of exposed symptomatic infected patients requiring hospitalization on the notified doctor's opinion.

\subsection{Recovered $R(t)$}

It is the symptomatic and asymptomatic infected who are recovered.

\section{Equilibirum Points and Stability Analysis}

\subsection{Positivity and Boundedness of the Solution for the Model(1)}

This subsection is provided to prove the positivity and boundedness of solution of the system (1) with initial (S(0), E(0), I(0), A(0), H(0), R(0)) $)^{\mathrm{T}} \in \mathbf{R}^{6}$.

This research process for points of stability is much more detailed in [16] [17].

For the above system (1), we find a region of attraction which is given by Lemma 1. 
Lemma 1 [18]

Suppose $\Omega \subset \mathbf{R} \times \mathbf{C}^{n}$ is open, $f_{i} \in(\Omega, \mathbf{R}), \quad i=1,2,3, \cdots, n$.

If $\left.f_{i}\right|_{x_{i}(t)=0, X_{t} \in \mathbf{C}^{n}} \geq 0, \quad X_{t}=\left(x_{1 t}, x_{2 t}, \cdots, x_{n t}\right)^{\mathrm{T}}, \quad i=1,2,3, \cdots, n$, then $\mathbf{C}^{n}$ $\left\{\phi=\left(\phi_{1}, \cdots, \phi_{n}\right): \phi \in \mathbf{C}\left([-\tau, 0], \mathbf{R}_{+}^{n}\right)\right\}$ is the invariant domain of the following equations

$$
\frac{\mathrm{d} x_{i}(t)}{\mathrm{d} t}=f_{i}\left(t, X_{t}\right), \quad t \geq \sigma, \quad i=1,2, \cdots, n
$$

where $\mathbf{R}_{+}^{n}=\left\{\left(x_{1}, \cdots, x_{n}\right): i=1, \cdots, n\right\}$.

Proposition 1 The system (1) is invariant in $\mathbf{R}_{+}^{6}$.

Proof. We have by the system (1):

$$
\begin{gathered}
\frac{\mathrm{d} X}{\mathrm{~d} t}=F(X(t)), X(0)=X_{0} \\
F(X(t))=\left(F_{1}(X), F_{2}(X), \cdots, F_{6}(X)\right)^{\mathrm{T}}
\end{gathered}
$$

We note that

$$
\begin{gathered}
\left.\frac{\mathrm{d} S}{\mathrm{~d} t}\right|_{S=0}=\Lambda \geq 0,\left.\frac{\mathrm{d} E}{\mathrm{~d} t}\right|_{E=0}=\beta S \frac{I+A}{N(t)} \geq 0,\left.\frac{\mathrm{d} I}{\mathrm{~d} t}\right|_{I=0}=(1-p) \varepsilon \geq 0 \\
\left.\frac{\mathrm{d} A}{\mathrm{~d} t}\right|_{A=0}=p \varepsilon E \geq 0,\left.\frac{\mathrm{d} H}{\mathrm{~d} t}\right|_{H=0}=(1-p) \varepsilon E \geq 0,\left.\frac{\mathrm{d} R}{\mathrm{~d} t}\right|_{R=0}=\left(\gamma_{1}+\tau\right) I(t)+\gamma_{2} A(t) \geq 0
\end{gathered}
$$

Then it follows from the Lemma 1 that $\mathbf{R}_{+}^{6}$ is an invariant set.

Lemma 2 The system (1) is bounded in the region

$$
\Omega=\left\{(S, E, I, A, H, R) \in \mathbf{R}_{+}^{6} \mid S+E+I+A+H+R \leq \Lambda\right\}
$$

The proof of Lemma 2 is obvious since we observed from the system that

$$
\begin{gathered}
\\
\text { which } \Rightarrow \lim _{t \rightarrow+\infty} \sup N(t) \leq \Lambda
\end{gathered}
$$

\subsection{Local Stability of Disease-Free Equilibrium (DFE)}

The DFE of our model (1) is given by:

$$
\Omega_{0}=\left(S_{0}, E_{0}, I_{0}, A_{0}, H_{0}, R_{0}\right)=(\Lambda, 0,0,0,0,0)
$$

The local stability of $\Omega_{0}$ can be established on the system (1) by using the next generation operator method.

The basic reproduction number $R_{0}$, the expected number of secondary infections produced by an index case in a completely susceptible population, is a key factor used in estimating the transmissibility of infectious disease.

For our model (1), the basic reproduction number is:

$$
R_{0}=\beta\left(\frac{q}{\varepsilon}+\frac{p}{\gamma_{1}}+\frac{1-p}{\gamma_{2}}\right)
$$

For more details on the calculation method of $R_{0}$, [19] [20] [21].

The threshold quantity $R_{0}$ is the basic reproduction number of the disease. 
This represents the average number of secondary cases generated by an infected person in a fully susceptible population. The epidemiological significance is that when $R_{0}$ is less than unity, a low number of infected individuals in the population will not cause major epidemics and so much disease to go away over time.

It should also be noted that $R_{0}$ is by no means a characteristic of a given disease, but the one of a given disease in a given host population. As a result, the same disease could have two different $R_{0}$ in two different host populations and two different diseases could also have two different $R_{0}$ in the same host population.

The effective reproduction number $R_{t}$ is defined as the expected number of secondary infections per infectious person in a population composed of both susceptible and non-susceptible hosts [22].

The formula for calculating $R_{t}$ is as follows:

$$
R_{t}=R_{0} \times \hat{s}(t)
$$

where $\hat{s}(t)$ is the fraction of the host population sensitive to time $t$.

If:

- $R_{t}>1$ : the number of new cases will be increased;

- $R_{t}=1$ : the disease will become endemic;

- $R_{t}<1$ : there will be a decrease in the number of new cases.

Lemma 3 The DFE, $\Omega_{0}$ of the model (1) is locally-asymptotically stable if $\Omega$ whenever $R_{t}>1$, and unstable if $R_{t}<1$.

\section{Estimation of Parameters and Results}

\subsection{Description of the Epidemiological Situation in the Central African Republic}

The data were observed over the period from March 03, 2020 to June 07, 2020, 14,259 samples were taken and tested with real-time PCR in the laboratory.

We have:

- 1570 positives, therefore a national prevalence over the period of $11 \%$;

- 5 epidemic peaks observed (31 to 24 April, 49 to 09 May, 80 to 16 May, 119 to 30 May, 163 to 06 June);

- The dynamics of the epidemic are nolinear.

The first case of COVID-19 confirmed on March 14, 2020 in the country was a case imported from Italy. As a result, other cases started to be reported. The Ministry of Public Health has therefore decided to systematically test all people arriving by land in Central African territory since the airport was already closed during the period.

It is therefore from these data that we estimated certain biological parameters in our mathematical model which is a non-linear model of the dynamics and circulation control of COVID-19 in the Central African Republic.

The general population is not quarantined during this period compared to other countries given the country's very weak economic context. Only barrier measures and social distancing have been adopted by the Ministry of Public 
Health and the population has been widely made aware of these various measures.

The data that we used to build our mathematical model are the data for the period from March 03, 2020 to June 07, 2020 retrieved from the World Health Organization website

(http://www.who.int/fr/emergencies/diseases/novel-coronavirus-2019).

In Figure 1, the cumulative curve weighs on the data for epidemiological weeks, this fact that we do not visualize very well the number of people who have died from COVID-19 (May 19, 2020, date of the first case of mortality linked to COVID-19, then May 29, 2020 and June 04, 2020) over the entire period of our study.

\subsection{Estimation of Parameters}

In order to fit the model to time series data from confirmed COVID-19 cases, it is necessary to define certain parameter values. In our model, birth and natural death rates were assumed to ignore over our study period. It is also assumed that the case distribution profile in the different provinces is similar across the country.

Table 1 describes the epidemiological dataset on COVID-19 in the Central African Republic. From these data, we estimated the parameters of our model (1).

We can clearly see in Table 1 that the prevalence of the COVID-19 pandemic is $11 \%$ in just 4 months.

Figure 2 shows the number of new cases confirmed by the Rt-PCR technique in the laboratory. The very first COVID-19 case in the country was detected on March 16, 2020.
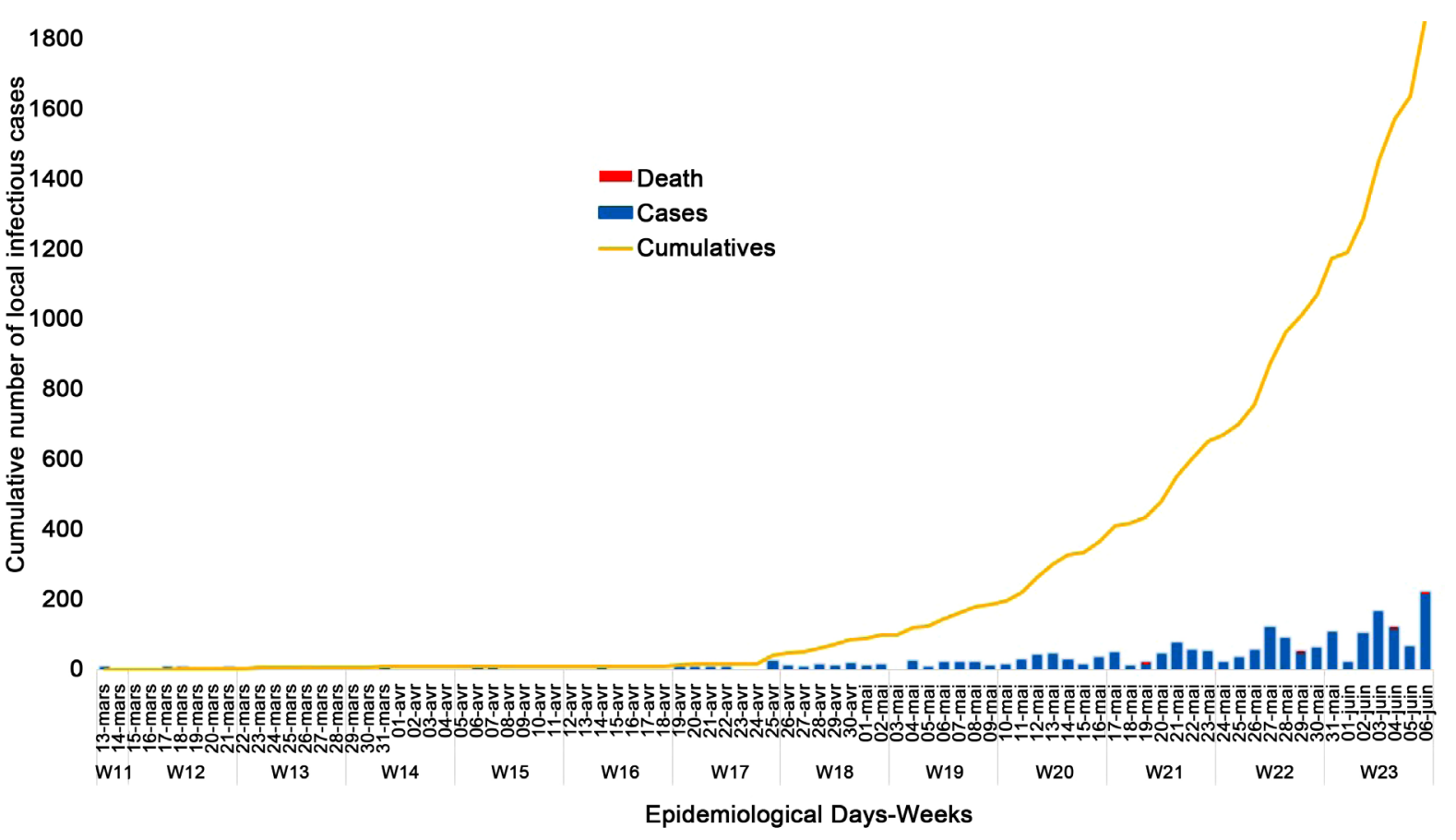

Figure 1. Cumulative number of local infectious cases COIVID-19. 
COVID-19 daily cases in Central African

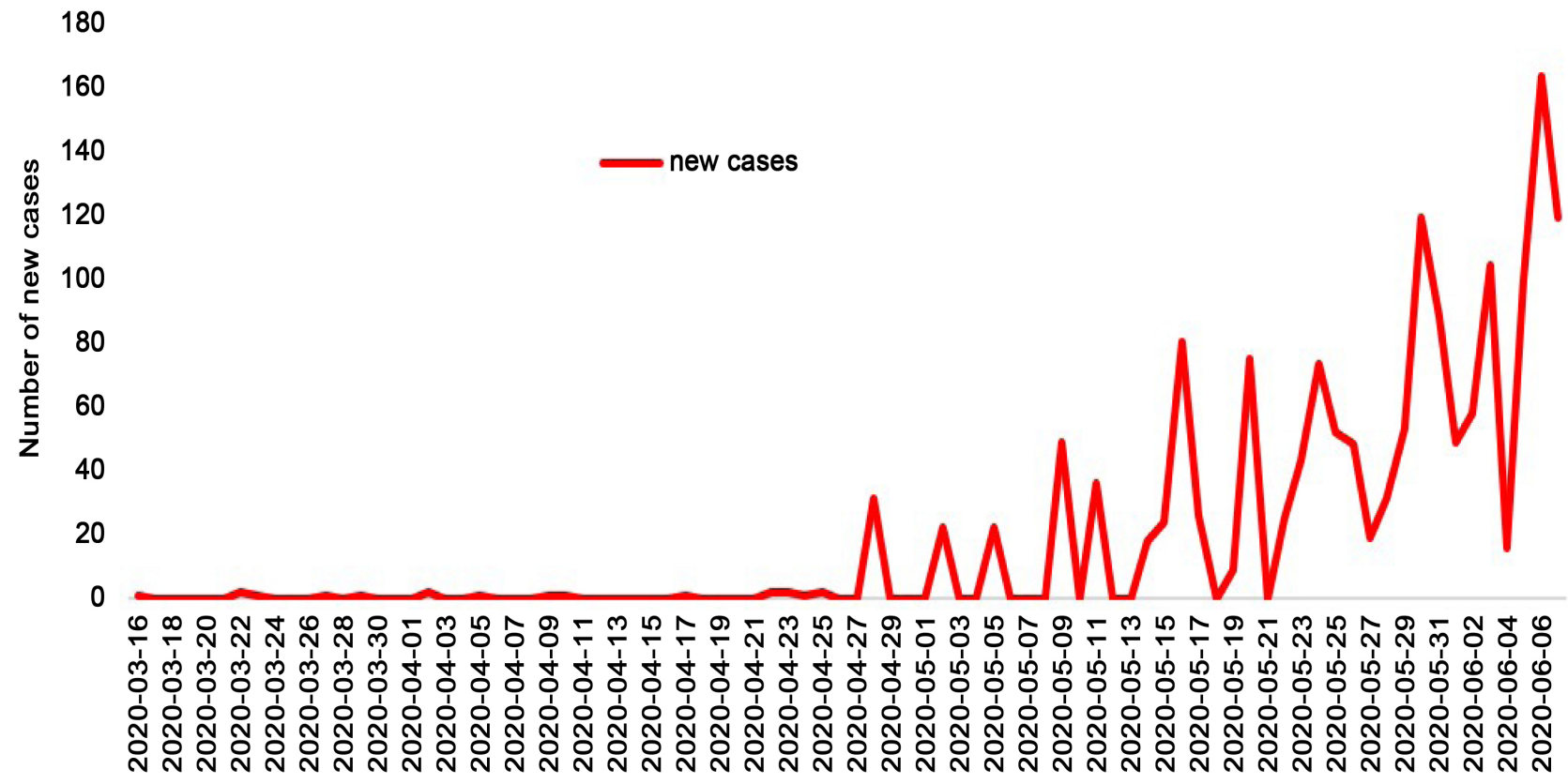

Figure 2. Number of confirmed COVID-19 cases PCR.

Table 1. COVID-19 data from March 16 to June 06, 2020.

\begin{tabular}{cc}
\hline Data & Values \\
\hline Total number samples & 14,259 \\
Number infected & 1570 \\
Number revered & 29 \\
Number of death & 5 \\
\hline
\end{tabular}

We used the maximum likelihood method to estimate the parameters of our model [23] for Table 2.

The force of infection $\beta=-q * \log (1-p)$ with $p$ the probability of infection during infectious contact (intrinsic property of the disease).

And $q=1-c$ where $c$ control of contact between individuals. We therefore evaluate the different values of $R_{0}$ for different values of $c$ (weak, medium and strong).

\subsection{Analytical Results}

We used R software with $R_{0}$ package to estimate reproduction numbers for epidemic outbreaks.

Assuming that there is no difference between cure rates in symptomatic and asymptomatic we have:

If we assume that there is a difference between the cure rates in symptomatic and asymptomatic patients, we have: 
Table 2. Estimates of biological parameters.

\begin{tabular}{ccc}
\hline Parameters & Biological Meaning & Estimated Values (95\% CI) \\
\hline$\Lambda$ & Recruitment rate of population & $0.87(0.86-0.89)$ \\
$\tau$ & COVID-19 death rate & $0.0032(0.0004-0.006)$ \\
$\gamma_{1}$ & symptomatic infection rate & $0.17(0.15-0.19)$ \\
$\gamma_{2}$ & asymptomatic infection rate & $0.83(0.81-0.85)$ \\
\hline
\end{tabular}

Table 3. Estimate of $R_{0}$.

\begin{tabular}{cccc}
\hline$c$. control & $q=1-c$ & $\beta$ & $R_{0}$ \\
\hline 0.25 & 0.75 & 0.22 & 1.45 \\
0.5 & 0.5 & 0.15 & 0.89 \\
0.85 & 0.15 & 0.04 & 0.23 \\
\hline
\end{tabular}

Table 4. Estimate of $R_{0}$ with difference in cure rate.

\begin{tabular}{cccc}
\hline$c$ control & $q=1-c$ & $\beta$ & $R_{0}$ \\
\hline 0.25 & 0.75 & 0.22 & 0.72 \\
0.5 & 0.5 & 0.15 & 0.4 \\
0.85 & 0.15 & 0.04 & 0.09 \\
\hline
\end{tabular}
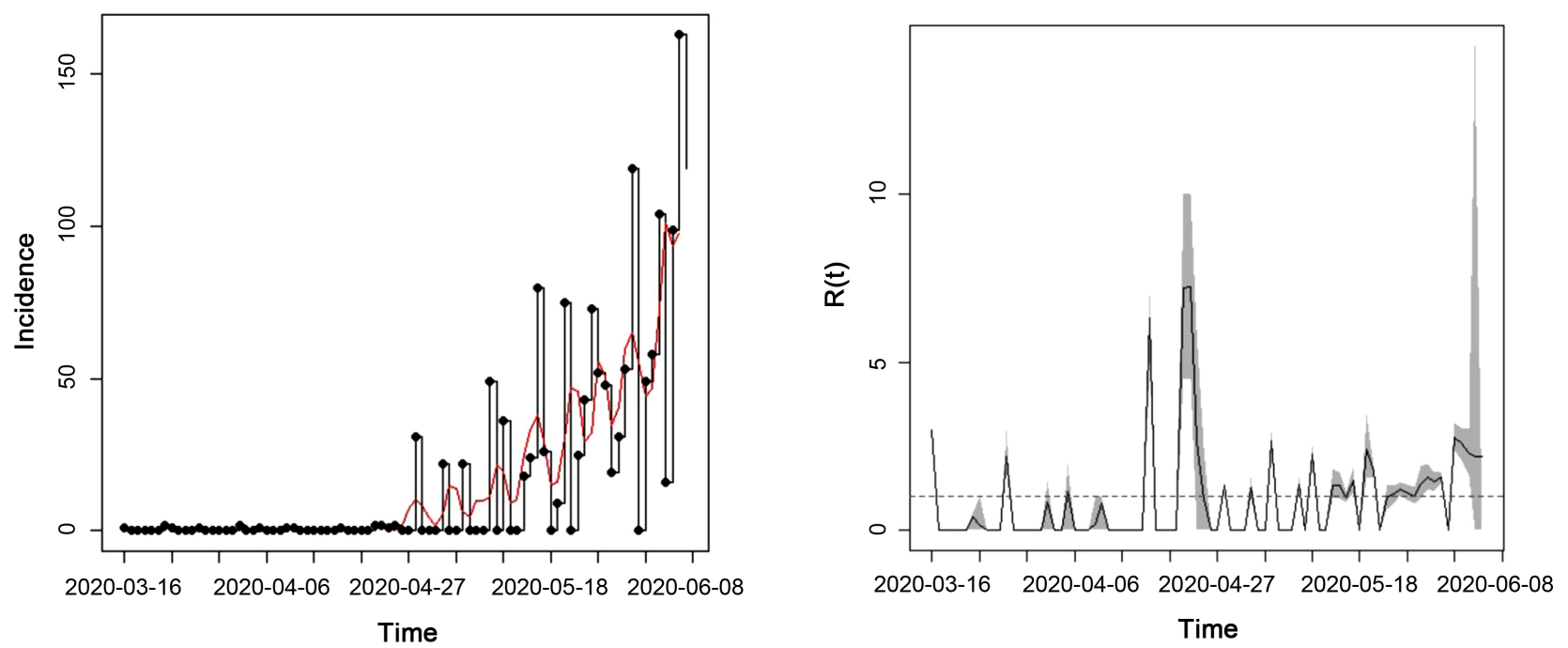

Figure 3. Epidemic curve and reproduction number (Time-Dependnet).

\section{Discussion}

COVID-19 is a pandemic that strikes all countries in the world. Each country controls this disease according to the means it has but also internationally recognized barrier gestures. During the study period, the Central African Government had also taken decisions going in the direction of social distancing and masking. We must recognize that if we really have to evaluate these different measures in the population, especially in Bangui, we cannot even reach $25 \%$.

This means that very few of the people of Bangui respect these social distancing 
and masking. This leads, according to Table 3, for a weak control or non-compliance with the social distancing and masking, the value of $\beta=0.22$ and $R_{0}=1.45>1$. Then the epidemic will continue in the population.

On the other hand, if the respect of social distancing and masking by the population reaches at least 50\%, the value of $\beta=0.15$ and $R_{0}=0.85<1$, the disease will die out over time. Indeed, our study goes well with the reality over the study period. At the start of the pandemic in the country, the social distancing and masking decided by the Government were not respected at all. It took a lot of awareness, and the squaring of major roads by the police by controlling buses and motorcycle taxis made it possible to control the pandemic well despite the limited resources of the Government.

In Table 4, we have made the scenario on the cure rate of symptomatic and asymptomatic, assuming that the cure rate in asymptomatic is higher than symptomatic. And there, we see that if the cure rate of asymptomatic patients is very high, the epidemic stabilizes.

Figure 3 shows the incidence and the number of reproduction $R_{t}$ in real time over the study period.

It must also be recognized that the low density unevenly spread and the low frequency of displacement of the Central African population played in its favor. Since over this period, there is a low frequency of displacement, unlike in countries with high density and frequency of displacement of the population.

\section{Conclusions}

In this paper, our objective is to estimate the number of reproduction in real time $R_{t}$ in retrospective and to study the measures of social distancing and masking controls. We have found that indeed by contact control through social distancing and masking remains the best possibility to control the COVID-19 pandemic. In addition, the high population density and population dynamics are an important factor in the spread of the disease.

We have shown that a strong contact control and the frequency of displacement of the population would reduce the evolution of the epidemic.

Therefore, for countries with low income and low density and frequency of population displacement, social distancing and masking would be the best possibility to control COVID-19.

\section{Acknowledgements}

Sincere thanks to the AM members for their professional performance, and a special thank you to the editorial members for a rare high quality attitude.

\section{Conflicts of Interest}

The authors declare that they have no conflict of interest.

\section{References}

[1] Allen, L. (1994) Some Discrete-Time SI, SIR, and SIS Epidemic Models. Mathemat- 
ical Biosciences, 124, 83-105. https://doi.org/10.1016/0025-5564(94)90025-6

[2] Cai, Y., Kang, Y., Banerjee, M. and Wang (2016) A Stochastic Epidemic Model Incorporating Media Coverage. Communications in Mathematical Sciences, 14, 893-910. https://doi.org/10.4310/CMS.2016.v14.n4.a1

[3] Yambiyo, B.M., Norouzi, F. and N'Guérékata, G.M. (2021) A Study an Epidemic SIR vis Homotopy Analysis Method in the Sense Caputo-Fractional System. Studies in Evolution Equations and Related Topics STEAM-H Series, Springer, Berlin.

[4] Tolles, J. and Luong, T. (2020) Modeling Epidemics with Compartmental Models. JAMA, 323, 2515-2516. https://doi.org/10.1001/jama.2020.8420

[5] Hethcote, W. (2000) The Mathematics of Infectious Diseases. SIAM Review, 42, 599-653. https://doi.org/10.1137/S0036144500371907

[6] Brauer, F. and Castillo-Chavez, C. (2019) Mathematical Models in Population Biology and Epidemiology. Texts in Applied Mathematics 69, Part of Springer Nature.

[7] Kucharski, J., et al. (2020) Early Dynamics of Transmission and Control of Covid-19: A Mathematical Modelling Study. The Lancet Infectious Diseases, 20, 553-558. https://doi.org/10.1016/S1473-3099(20)30144-4

[8] Majumder, A., Bala, T.K., Adak, D., N’Guérékata, G.M. and Bairagi, N. (2020) Evaluating the Current Epidemiological Status of Italy: Insights from a Stochastic Epidemic Model. Nonlinear Studies, 27, 1169-1177.

[9] Read, M., Bridgen, J.R., Cummings, D., Ho, A. and Jewell, C.P. (2020) Novel Coronavirus 2019-Ncov: Early Estimation of Epidemiological Parameters and Epidemic Predictions. https://doi.org/10.1101/2020.01.23.20018549

[10] Peng, L., Yang, W., Zhang, D., Zhuge, C. and Hong, L. (2020) Epidemic Analysis of Covid-19 in China by Dynamical Modeling.

https://doi.org/10.1101/2020.02.16.20023465

[11] Shen, M., Peng, Z., Xiao, Y. and Zhang, L. (2020) Modelling the Epidemic Trend of the 2019 Novel Coronavirus Outbreak in China.

https://doi.org/10.1101/2020.01.23.916726

[12] Jewell, P., Lewnard, J.A. and Jewell, B.L. (2020) Predictive Mathematical Models of the COVID-19 Pandemic. JAMA, 323, 1893-1894. https://doi.org/10.1001/jama.2020.6585

[13] Nadim, S., et al. (2020) Short-Term Predictions and Prevention Strategies for Covid-2019: A Model Based Study.

[14] Aronna, M., Guglielmi, R. and Moschen, L.M. (2021) A Model for COVID-19 with Isolation, Quarantine and Testing as Control Measures. Epidemics, 34, Article ID: 100437. https://doi.org/10.1016/j.epidem.2021.100437

[15] Zongo, P.M., et al. (2020) A Model of Covid-19 Transmission to Understand the Effectiveness of the Containment Measures: Application to Data from France. Epidemiology and Infection, 148, e221. https://doi.org/10.1017/S0950268820002162

[16] Sardar, T., Ghosh, I., Xavier, R. and Chattopadhyay, J. (2020) A Realistic Two-Strain Model for Mers-Cov Infection Uncovers the High Risk for Epidemic Propagation. PLOS Neglected Tropical Diseases, 14, e0008065. https://doi.org/10.1371/journal.pntd.0008065

[17] Singh, R. and Adhikari, R. (2020) Age-Structured Impact of Social Distancing on the Covid-19 Epidemic in India.

[18] Yang, X., Chen, L. and Chen, J. (1996) Permanence and Positive Periodic Solution for the Single-Species Nonautonomous Delay Diffusive Models. Computer Mathematics with Applications, 32, 109-116. 
https://doi.org/10.1016/0898-1221(96)00129-0

[19] Den Driessche, P.V. and Watmough, J. (2002) Reproduction Numbers and Sub-Threshold Endemic Equilibria for Compartmental Models of Disease Transmission. Mathematical Biosciences, 180, 29-48.

https://doi.org/10.1016/S0025-5564(02)00108-6

[20] Diekmann, O., et al. (1990) On the Definition and the Computation of the Basic Reproductive Ratio $\mathrm{R}_{0}$ in Models for Infectious Diseases. Journal of Mathematical Biology, 35, 503-522. https://doi.org/10.1007/BF00178324

[21] Hyman, J.M. and Li, J. (2000) An Intuitive Formulation for the Reproduction Number for the Spread of Diseases in Heterogeneous Population. Mathematical Biosciences, 167, 65-86. https://doi.org/10.1016/S0025-5564(00)00025-0

[22] Rothman, K.J., Greenland, S. and Lash, T.L. (2008) Modern Epidemiology. Lippincott Williams and Wilkins, Philadelphia.

[23] Kuhn, E. and Lavielle, M. (2005) Maximum Likelihood Estimation in Nonlinear Mixed Effects Models. Computational Statistics \& Data Analysis, 49, 1177-1186. https://doi.org/10.1016/j.csda.2004.07.002 